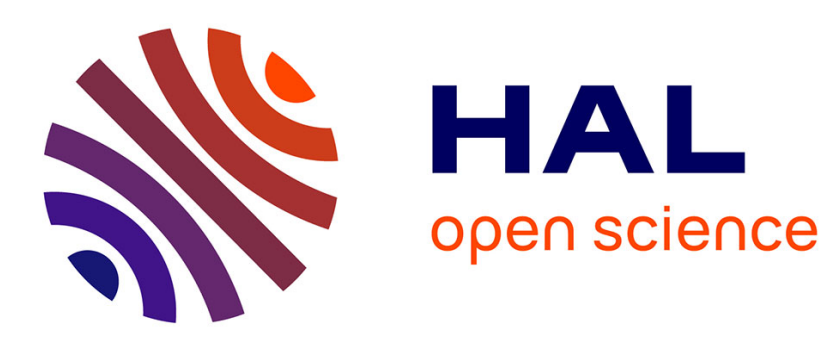

\title{
Optimal repeated measurement designs for a model with partial interactions
}

Pierre Druilhet, Walter Tinsson

\section{To cite this version:}

Pierre Druilhet, Walter Tinsson. Optimal repeated measurement designs for a model with partial interactions. Biometrika, 2009, 96 (3), pp.677-690. hal-00221433

\section{HAL Id: hal-00221433 \\ https://hal.science/hal-00221433}

Submitted on 28 Jan 2008

HAL is a multi-disciplinary open access archive for the deposit and dissemination of scientific research documents, whether they are published or not. The documents may come from teaching and research institutions in France or abroad, or from public or private research centers.
L'archive ouverte pluridisciplinaire HAL, est destinée au dépôt et à la diffusion de documents scientifiques de niveau recherche, publiés ou non, émanant des établissements d'enseignement et de recherche français ou étrangers, des laboratoires publics ou privés. 


\title{
Optimal repeated measurement designs for a model with partial interactions
}

\author{
Pierre Druilhet and Walter Tinsson \\ CREST-ENSAI and Université de Pau
}

\begin{abstract}
We consider circular crossover designs for models with partial interactions: we assume that the carryover effect of a treatment on itself is different from the carryover effect on other treatments. We generalize Kushner (1997) and Kunert and Martin (2000) methods to find optimal designs when the parameters of interest are total effects, which corresponds to the use of a single treatment, once selected. We give a lower bound of $\Phi_{p}$ criteria and propose efficient designs of small sizes.
\end{abstract}

AMS 2000 subject classifications. Primary 62K05, 62K10.

Key words and phrases. Crossover design, optimal design, universal optimality, total effects 


\section{Introduction}

In traditionnal crossover designs, the statistical model consider that each treatment has a carryover effect, which, for parsimony, is assumed not interacting with direct treatment effects. To enrich this model, Hedayat and Afsarinejad (2002) propose a model with partial interactions: the carryover effect of a treatment is different if the treatment is preceded by itself. For such model, Kunert and Stufken (2002) find that universally optimal designs for direct treatment effects have no consecutive pairs of identical treatments. This feature is mainly due to the fact that partial interactions between carryover and direct treatment effects are considered as nuisance parameter. However, the aim of an experiment is to select a single treatment which will be used alone and then will be preceded by itself. In that case, the parameter of interest, called total effects, is the sum of direct treatments and self carryover effects. Bailey and Druilhet (2004) give a review of situations where total effects are considered in the literature. They show that neighbour balanced designs having no treatment preceded by itself are efficient when the number of periods is small. However, for models with partial interactions, such designs are inefficient because total effects are not estimable.

In this paper, we propose a general method to construct optimal circular 
crossover designs for total effects under models with partial interactions. Upper bounds of the information matrix are obtained by a new method that generalizes the approaches of Kushner (1997), Kunert and Martin (2000) and Druilhet and Bailey (2004). Then we show that, in most cases, optimal designs are generated by one sequence or a mixture of two sequences that belongs to a set of $k-1$ possible sequences, where $k$ is the number of periods. Finally, we give some examples of optimal designs for several values of $k$. We also propose efficient designs generated by only one sequence of treatments. To simplify our presentation, we first construct optimal design for a model without period effects. Then we obtain optimal designs for a model with period effects by generalizing Kunert (1983) results on orthogonal effects.

\section{The designs and the models}

Let $d(i, j) \in\{1, \ldots, t\}$ be the treatment assigned to subject $i$ in period $j$, $1 \leq i \leq b, 1 \leq j \leq k$. We assume that the response $y_{i j}$ follows the model:

$$
y_{i j}=\beta_{i}+\tau_{d(i, j)}+\lambda_{d(i, j-1)}+\chi_{d(i, j-1) d(i, j)}+\varepsilon_{i j}
$$

where $\beta_{i}$ is the effect of subject $i, \tau_{i}$ is the effect of treatment $i, \lambda_{i}$ is the general carryover effect of treatment $i$ and $\chi_{i j}$ is the additional specific carryover effect when treatment $i$ is followed by itself $\left(\chi_{i j}=0\right.$ if $\left.i \neq j\right)$. The 
errors $\varepsilon_{i j}$ are assumed i.i.d. with expectation 0 and variance $\sigma^{2}$. The model with period effects is considered in Section 5. In vector notation, we have:

$$
Y=B \beta+T_{d} \tau+L_{d} \lambda+S_{d} \chi+\varepsilon
$$

where $B, T_{d}, L_{d}$ and $S_{d}$ are incidence matrices of subjects, direct treatments, carryover and specific self-carryover effects. Note that the parametrization, although equivalent, is slightly different from that of Hedayat and Afsarinejad (2002) or Kunert and Stufken (2002): in our model, the self carryover effect is equal to $\rho_{d(i, j-1)}+\chi_{d(i, j-1) d(i, j)}$. We define the vector $\phi$ of total effects by $\phi=\tau+\lambda+\chi$. It corresponds to the effect of a treatment when it is preceded by itself. Note that if $\theta^{\prime}=\left(\tau^{\prime}, \lambda^{\prime}, \chi^{\prime}\right)$ and $K^{\prime}=\left(I_{t}\left|I_{t}\right| I_{t}\right)$, then

$$
\phi=K^{\prime} \theta .
$$

Because the effect of having no treatment differs from the carryover effect of any treatment, we consider only designs with pre-periods, i.e. designs with one period, called pre-period, added at the beginning. On this preperiod, each subject receive a treatment but the response is not used in the analysis. We assume that the designs are circular, i.e. the treatment assigned to a subject in the pre-period is the same as the treatment assigned in the last period. The circularity condition may be written $d(i, 0)=d(i, k)$. We denote 
by $\Omega_{t, b, k}$ the set of all circular designs with $t$ treatments, $b$ subjects and $k$ periods.

\section{Some technical tools}

In this section, we present a general method to derive optimal crossover designs. We generalize and unify the techniques developed by Kushner (1997), Kunert and Martin (2000) and Bailey and Druilhet (2004).

Let denote by $\mathbb{1}_{k}, I_{k}$ and $J_{k}$ respectively the vector of ones of length $k$, the $(k, k)$ identity matrix and the $(k, k)$ matrix of ones. For any matrix $A$, denote by $A^{+}$the Moore-Penrose inverse of $A$. The projection matrix onto the column span of matrix $A$ is denoted by $\operatorname{pr}_{(A)}=A\left(A^{\prime} A\right)^{+} A^{\prime}$. We

denote $\operatorname{pr}_{(A)}^{\perp}=I-\operatorname{pr}_{(A)}$ and $Q_{k}=\operatorname{pr}_{\left(\mathbb{1}_{k}\right)}^{\perp}=I_{k}-k^{-1} J_{k}$. For a square matrix $A$, we denote by $\operatorname{tr}(A)$ the trace of $A$. For two symmetric matrices $M$ and $N, M \leq N$ means that $N-M$ is a nonnegative definite matrix (Loewner ordering). A matrix $M$ is completely symmetric if $M=a I+b J$ for some scalars $a$ and $b$.

\subsection{Information matrices and its extremal representation}

Consider a generic partitioned linear model:

$$
Y=A \alpha+B \beta+\varepsilon \quad \text { with } \quad \mathbb{E}(\varepsilon)=0 \quad \text { and } \operatorname{Var}(\varepsilon)=\sigma^{2} I,
$$


where $\alpha$ is a vector of length $q$. It is well known (see e.g. Kunert, 1983) that the information matrice $C[\alpha]$ of the parameter $\alpha$ is

$$
C[\alpha]=A^{\prime} \operatorname{pr}_{(B)}^{\perp} A .
$$

Consider now a subsystem $K^{\prime} \alpha$ where $K$ is a $(q, s)$ matrix. The information matrix $C\left[K^{\prime} \alpha\right]$ of $K^{\prime} \alpha$ may be defined by the extremal representation (Gaffke, 1987 or Pukelsheim, 1993):

$$
C\left[K^{\prime} \alpha\right]=\min _{L \in \mathbb{R}^{q \times s}: L^{\prime} K=I_{s}} L^{\prime} C[\alpha] L .
$$

where the minimum, taken relative to the Loewner ordering, exists and is unique. If $L^{*}$ is a $(q, s)$ matrix that minimizes $L^{\prime} C[\alpha] L$ under the constraint $L^{\prime} K=I_{s}$, then

$$
C\left[K^{\prime} \alpha\right]=L^{* \prime} C[\alpha] L^{*}
$$

Formula (5) will be central in the following mainly because it provides a linear relationship between $C[\alpha]$ and $C\left[K^{\prime} \alpha\right]$.

The main issue to construct optimal designs will be to find $L^{*}$ for designs candidate to optimality. Because a global minimum in (4) exists, $L^{*}$ minimizes $L^{\prime} C[\alpha] L$ iff $L^{*}$ minimizes $\operatorname{tr}\left(L^{\prime} C[\alpha] L\right)$. So, we have 


$$
\operatorname{tr}\left(C\left[K^{\prime} \alpha\right]\right)=\operatorname{tr}\left(L^{* \prime} C[\alpha] L^{*}\right)=\min _{L \in \mathbb{R}^{q \times s}: L^{\prime} K=I_{s}} \operatorname{tr}\left(L^{\prime} C[\alpha] L\right) .
$$

In our problem, the matrix $C[\alpha]$ will have a natural block structure with completely symmetric blocks. In that case, the following results show that the matrice $L^{*}$ have also completely symmetric blocks.

Proposition 1 Let $C[\alpha]=\left(\left(\mathcal{C}_{i j}\right)\right)_{i, j=1, \ldots, q}$ be a block matrix, where the blocks $\mathcal{C}_{i j}$ are $(t, t)$ completely symmetric matrices for $i=1, \ldots, q$ and $j=$ $1, \ldots, q$. Let $K^{\prime}=\left(K_{1}^{\prime}, \ldots, K_{q}^{\prime}\right)$ where for all $i, K_{i}$ are $(t, t)$ completely symmetric matrices. Then $C\left[K^{\prime} \alpha\right]$ is completely symmetric. Moreover, if $L^{* \prime}=\left(L_{1}^{* \prime}, \ldots, L_{q}^{* \prime}\right)$ satisfies both Eq. (5) and the constraint $L^{* \prime} K=I_{t}$, then $L_{i}^{*}, i=1, \ldots, q$, can be chosen completely symmetric.

Proof: The proof is given in Appendix A.

The interest of this result is that $L_{i}^{*}=a_{i}^{*} I_{t}+b_{i}^{*} J_{t}$ can be found out by minimizing $q\left(a_{1}^{*}, b_{1}^{*}, \ldots, a_{q}^{*}, b_{q}^{*}\right)=\operatorname{tr}\left(L^{* \prime} C[\alpha] L^{*}\right)$ which is a polynomial of second degree in $a_{1}^{*}, b_{1}^{*}, \ldots, a_{q}^{*}, b_{q}^{*}$.

Corollary 2 Under the notations and assumptions of Proposition 1, if moreover $\mathcal{C}_{i j} \mathbb{1}_{t}=0$ for $i=1, \ldots, q$ and $j=1, \ldots, q$, then $L_{i}^{*}$ can be chosen equal to $a_{i}^{*} I_{t}$ for some scalar $a_{i}^{*}$. 
Proof: By Proposition 1, $L_{i}^{*}$ can be chosen equal to $a_{i}^{*} I_{t}+b_{i}^{*} J_{t}$. Since, $\mathcal{C}_{i j} J_{t}=J_{t} \mathcal{C}_{i j}=0, L_{i}^{\prime} \mathcal{C}_{i j} L_{j}=a_{i}^{*} a_{j}^{*} \mathcal{C}_{i j}$ does not depends on $b_{i}^{*}$ and $b_{j}^{*}$ and the result follows.

The following lemma, although straightforward, is useful to calculate explicitly $L^{*}$.

Lemma 3 Let $C$ be a nonnegative $(u, u)$ matrix, $\gamma$ be a k-vector, and $\gamma \mapsto$ $L_{\gamma}$ be a linear mapping with $L_{\gamma} a(u, v)$ matrix. Then, $q(\gamma)=\operatorname{tr}\left(L_{\gamma}^{\prime} C L_{\gamma}\right)$ is a multivariate convex quadratic polynomial in $\gamma_{1}, \ldots, \gamma_{k}$. Moreover, $q\left(\gamma^{*}\right)$ is a minimum of $q(\gamma)$ if and only if $\frac{\partial q}{\partial \gamma}\left(\gamma^{*}\right)=0$.

\subsection{Some examples}

Under the notations and assumptions of Proposition 1 and Corollary 2, i.e. assuming that $\mathcal{C}_{i j}$ are completely symmetric and that $\mathcal{C}_{i j} \mathbb{1}_{t}=0$, we show in some cases how the matrices $L^{*}$ can be obtained. The three first examples give new presentations of known results. The last one will be used in this paper. We denote $c_{i j}=\operatorname{tr}\left(\mathcal{C}_{i j}\right)$.

Example 1 : let $q=2$ and $K^{\prime}=\left(I_{t} \mid 0\right)$. By Corollary 2 and because $L^{* \prime} K=I_{t}, L^{* \prime}$ can be chosen to be equal to $\left(I_{t} \mid x^{*} I_{t}\right)$ for some $x^{*} \in \mathbb{R}$. By 
Eq. (6), $x^{*}$ can be found out by minimizing

$$
q(x)=\operatorname{tr}\left(L^{\prime} C[\alpha] L\right)=c_{11}+2 x c_{12}+x^{2} c_{22} .
$$

This quadratic function was used by Kushner (1997, Eq. 4.1). The minimum is obtained for $x^{*}=\frac{c_{12}}{c_{22}}$ and we found $C\left[K^{\prime} \alpha\right]=\mathcal{C}_{11}-\mathcal{C}_{12} \mathcal{C}_{22}^{+} \mathcal{C}_{21}$, the Schurcomplement of $C_{22}$.

Example 2: let $q=3$ and $K^{\prime}=\left(I_{t}|0| 0\right)$. As in example $1, L^{* \prime}$ can be chosen equal to $\left(I_{t}\left|x^{*} I_{t}\right| y^{*} I_{t}\right)$, where $x^{*}$ and $y^{*}$ minimize the quadratic function

$$
q(x, y)=\operatorname{tr}\left(L^{\prime} C[\alpha] L\right)=c_{11}+x^{2} c_{22}+y^{2} c_{33}+2 x c_{12}+2 y c_{13}+2 x y c_{23} .
$$

This quadratic function was used by Kunert and Martin (2000, Proposition $3)$.

Example 3: let $q=2$ and $K^{\prime}=\left(I_{t} \mid I_{t}\right)$. We have $L^{* \prime}=\left(x^{*} I_{t} \mid\left(1-x^{*}\right) I_{t}\right)$, where $x^{*}$ minimizes

$$
q(x)=x^{2} c_{11}+(1-x)^{2} c_{22}+2 x(1-x) c_{12} .
$$

If $\mathcal{C}_{11}=\mathcal{C}_{22}$, then $x^{*}=\frac{1}{2}$ and $L^{*}=\frac{1}{2} K$. Therefore, $C\left[K^{\prime} \alpha\right]=\frac{1}{4} K^{\prime} C[\alpha] K$. This equation was obtained in a different way by Bailey and Druilhet (2004) in order to construct optimal designs for total effects under models without interaction. 
Example 4: let $q=3$ and $K^{\prime}=\left(I_{t}\left|I_{t}\right| I_{t}\right)$. We assume that $\mathcal{C}_{11}=\mathcal{C}_{22}$ and that $\mathcal{C}_{13}=\mathcal{C}_{23}$. We no longer assume that $\mathcal{C}_{33} \mathbb{1}_{t}=0$. We have $L^{* \prime}=$ $\left(x_{1}^{*} I_{t}\left|x_{2}^{*} I_{t}\right|\left(1-x_{1}^{*}-x_{2}^{*}\right) I_{t}+y^{*} J_{t}\right)$, where $x_{1}^{*}, x_{2}^{*}$ and $y^{*}$ minimize

$$
\begin{aligned}
q\left(x_{1}, x_{2}, y\right)= & \left(x_{1}^{2}+x_{2}^{2}\right) c_{11}+2\left(x_{1}+x_{2}\right) c_{11}-\left(x_{1}+x_{2}\right)^{2}\left(2 c_{13}+c_{33}\right) \\
& +2\left(x_{1}+x_{2}\right)\left(c_{13}-c_{33}-\widetilde{c}_{33}\right) \\
& +2 y \widetilde{c}_{33}+t y^{2} \widetilde{c}_{33}+c_{33}
\end{aligned}
$$

with $\widetilde{c}_{33}=\operatorname{tr}\left(J_{t} \mathcal{C}_{33}\right)=t^{-1} \operatorname{tr}\left(J_{t} \mathcal{C}_{33} J_{t}\right)$. Note that $L^{*}$ does not satisfy $L^{* \prime} K=$ $I_{t}$. This is in fact a simplified form of

$$
\widetilde{L}^{* \prime}=\left(x_{1}^{*} I_{t}+u^{*} J_{t}\left|x_{2}^{*} I_{t}-\left(u^{*}+v^{*}\right) J_{t}\right|\left(1-x_{1}^{*}-x_{2}^{*}\right) I_{t}+y^{*} J_{t}\right),
$$

noting that the terms $u^{*} J_{t}$ and $\left(u^{*}+v^{*}\right) J_{t}$ vanish in the expression of $\widetilde{L}^{*} C[\alpha] \widetilde{L}^{*}$. By lemma 3 and by symmetry of $q\left(x_{1}, x_{2}, y\right)$ in $x_{1}, x_{2}$, it is easy to see that $x_{1}^{*}$ and $x_{2}^{*}$ can be chosen equal. Denote $x^{*}=x_{1}^{*}=x_{2}^{*}$. From Eq. (7), $x^{*}$ and $y^{*}$ can be found out by minimizing

$$
\begin{aligned}
q(x, y)= & c_{33}+4\left(c_{13}-c_{33}\right) x+2 \widetilde{c}_{33} y+2\left(c_{11}+c_{12}-4 c_{13}+2 c_{33}\right) x^{2} \\
& +t \widetilde{c}_{33} y^{2}-4 \widetilde{c}_{33} x y
\end{aligned}
$$

and the minimum $q\left(x^{*}, y^{*}\right)$ is equal to $\operatorname{tr}\left(C\left[K^{\prime} \alpha\right]\right)$. 


\section{Optimal circular crossover designs for total ef- \\ fects}

From Kiefer (1975), a design $d^{*}$ for which the information matrix $C_{d^{*}}[\phi]$ is completely symmetric and that maximizes the trace of $C_{d}[\phi]$ over all the designs $d$ in $\Omega_{t, b, k}$ is universally optimal. In this section, we propose a method to construct universal optimal design for total effects.

\subsection{Upper bound of $\operatorname{tr} C_{d}[\phi]$}

For a design $d$, the information matrix for the whole parameter $\theta^{\prime}=\left(\tau^{\prime}, \lambda^{\prime}, \chi^{\prime}\right)$ in Model (1) is:

$$
C_{d}[\theta]=\left(\begin{array}{ccc}
T_{d}^{\prime} \operatorname{pr}_{(B)}^{\perp} T_{d} & T_{d}^{\prime} \operatorname{pr}_{(B)}^{\perp} L_{d} & T_{d}^{\prime} \operatorname{pr}_{(B)}^{\perp} S_{d} \\
L_{d}^{\prime} \operatorname{pr}_{(B)}^{\perp} T_{d} & L_{d}^{\prime} \operatorname{pr}_{(B)}^{\perp} L_{d} & L_{d}^{\prime} \operatorname{pr}_{(B)}^{\perp} S_{d} \\
S_{d}^{\prime} \operatorname{pr}_{(B)}^{\perp} T_{d} & S_{d}^{\prime} \operatorname{pr}_{(B)}^{\perp} L_{d} & S_{d}^{\prime} \operatorname{pr}_{(B)}^{\perp} S_{d}
\end{array}\right)=\left(\begin{array}{ccc}
\mathcal{C}_{d 11} & \mathcal{C}_{d 12} & \mathcal{C}_{d 13} \\
\mathcal{C}_{d 12}^{\prime} & \mathcal{C}_{d 22} & \mathcal{C}_{d 23} \\
\mathcal{C}_{d 13}^{\prime} & \mathcal{C}_{d 23}^{\prime} & \mathcal{C}_{d 33}
\end{array}\right) .
$$

By circularity of the designs, $B^{\prime} T_{d}=B^{\prime} L_{d}, T_{d}^{\prime} T_{d}=L_{d}^{\prime} L_{d}$ and $T_{d}^{\prime} S_{d}=$ $L_{d}^{\prime} S_{d}=S_{d}^{\prime} S_{d}=S_{d}^{\prime} L_{d}=S_{d}^{\prime} T_{d}$ and therefore $\mathcal{C}_{d 22}=\mathcal{C}_{d 11}$ and $\mathcal{C}_{d 23}=\mathcal{C}_{d 13}$. As in Section 3.2, we define $c_{d i j}=\operatorname{tr}\left(\mathcal{C}_{d i j}\right)$ and $\widetilde{c}_{d i j}=\operatorname{tr}\left(J \mathcal{C}_{d i j}\right)$.

A design is said to be symmetric if all the blocks $C_{d i j}$ are completely symmetric, or equivalently, if $C_{d}[\theta]$ is invariant by any permutations of the treatment labels. 
Proposition 4 For any design d, the information matrix for total effects $\phi$ satisfies:

$$
\operatorname{tr} C_{d}[\phi] \leq \min _{x, y} q_{d}(x, y)
$$

where $q_{d}(x, y)$ is defined by (8). Equality holds if $C_{d i j}[\theta]$ are completely symmetric for $1 \leq i \leq 3$ and $1 \leq j \leq 3$.

Proof : For a design $d$, we denote $\bar{C}_{d}[\theta]=\frac{1}{t !} \sum_{\sigma \in \mathcal{S}_{t}}\left(I_{3} \otimes P_{\sigma}\right) C_{d}[\theta]\left(I_{3} \otimes P_{\sigma}^{\prime}\right)$ the symmetrized information matrix for $\theta$. By conctruction, $\bar{C}_{d i j}$ are completely symmetric for all $i$ and $j$. Moreover, $c_{d i j}=\operatorname{tr}\left(C_{d i j}\right)=\operatorname{tr}\left(\bar{C}_{d i j}\right)$ and $\widetilde{c}_{d i j}=\operatorname{tr}\left(J_{t} C_{d 33}\right)=\operatorname{tr}\left(J_{t} \bar{C}_{d 33}\right)$. Denote by $\bar{C}_{d}[\phi]$ the information matrix of $\phi$ associated to $\bar{C}_{d}[\theta]$. By concavity of $C_{d}[\phi]$ w.r.t. $C_{d}[\theta]$, (see Pukelsheim, 1993 p. 77$), C_{d}[\phi] \leq \bar{C}_{d}[\phi]$ and $\operatorname{tr} C_{d}[\phi] \leq \min _{x, y} q_{d}(x, y)$.

A design is called degenerate if $\widetilde{c}_{d 33}=\operatorname{tr}\left(J_{t} C_{d 33}[\theta]\right)=0$ or equivalently if each block either contains only one treatment or has no treatment preceded by itself. Note that the information matrix of a degenerate design is null and therefore such a design is not considered. The following lemma shows that the minimization of $q_{d}(x, y)$ may be reduce to the minimization of a one variable quadratic function.

Lemma 5 Let $d$ be a non-degenerate design. The values $x^{*}$ and $y^{*}$ that 
minimize $q_{d}(x, y)$ satisfy $2 x^{*}-t y^{*}-1=0$. Moreover, $x^{*}$ minimizes $q_{d}(x)$, where

$q_{d}(x)=c_{d 33}-\frac{1}{t} \widetilde{c}_{d 33}+4\left(c_{d 13}-c_{d 33}+\frac{1}{t} \widetilde{c}_{d 33}\right) x+2\left(c_{d 11}+c_{d 12}-4 c_{d 13}+2 c_{d 33}-\frac{2}{t} \widetilde{c}_{d 33}\right) x^{2}$,

and $q_{d}\left(x^{*}, y^{*}\right)=q_{d}\left(x^{*}\right)$.

Proof: by lemma $3, \frac{\partial q_{d}}{\partial y}\left(x^{*}, y^{*}\right)=0$ at the minimum. For a non-degenerate design, this is equivalent to $2 x^{*}-t y^{*}-1=0$. Replacing $y$ by $2(x-1) / t$, we found $q_{d}(x, y)=q_{d}(x)$. Therefore, $x^{*}$ necessarily minimizes $q_{d}(x)$.

\subsection{Decomposition over the blocks}

It is well known that $C_{d}[\theta]$ is the sum of the information matrices $C_{d u}$ corresponding to Block $u$. Denote by $T_{d u}, L_{d u}$ and $S_{d u}$ the incidence matrices for Block $u$. Thus, $T_{d}^{\prime}=\left(T_{d 1}^{\prime}|\ldots| T_{d b}^{\prime}\right), L_{d}^{\prime}=\left(L_{d 1}^{\prime}|\ldots| L_{d b}^{\prime}\right), S_{d}^{\prime}=$ $\left(S_{d 1}^{\prime}|\ldots| S_{d b}^{\prime}\right)$ and:

$$
C_{d}[\theta]=\sum_{u=1}^{b} C_{d u}[\theta]=\sum_{u=1}^{b}\left(\begin{array}{ccc}
T_{d u}^{\prime} Q_{k} T_{d u} & T_{d u}^{\prime} Q_{k} L_{d u} & T_{d u}^{\prime} Q_{k} S_{d u} \\
L_{d u}^{\prime} Q_{k} T_{d u} & L_{d u}^{\prime} Q_{k} L_{d u} & L_{d u}^{\prime} Q_{k} S_{d u} \\
S_{d u}^{\prime} Q_{k} T_{d u} & S_{d u}^{\prime} Q_{k} L_{d u} & S_{d u}^{\prime} Q_{k} S_{d u}
\end{array}\right)
$$


We decompose in the same way $\operatorname{tr}\left(\mathcal{C}_{d i j}\right)$ and $\operatorname{tr}\left(\mathcal{C}_{d i j} J_{t}\right)$ :

$$
c_{d i j}=\operatorname{tr}\left(\mathcal{C}_{d i j}\right)=\sum_{u=1}^{b} c_{d i j}^{(u)} \text { and } \widetilde{c}_{d i j}=\operatorname{tr}\left(\mathcal{C}_{d i j} J_{t}\right)=\sum_{u=1}^{b} \widetilde{c}_{d i j}^{(u)}
$$

denoting by $c_{d i j}^{(u)}$ and by $\widetilde{c}_{d i j}^{(u)}$ the contributions of Block $u$. For example, for $i=j=1$ we have:

$$
c_{d 11}=\operatorname{tr}\left(\mathcal{C}_{d 11}\right)=\operatorname{tr}\left(\sum_{u=1}^{b} T_{d u}^{\prime} Q_{k} T_{d u}\right)=\sum_{u=1}^{b} c_{d 11}^{(u)} \text { with } c_{d 11}^{(u)}=\operatorname{tr}\left(T_{d u}^{\prime} Q_{k} T_{d u}\right) .
$$

Simplifications of these forms give:

$$
c_{d 11}^{(u)}=k-\frac{n_{u}^{s}}{k}, c_{d 12}^{(u)}=m_{u}-\frac{n_{u}^{s}}{k}, c_{d 13}^{(u)}=m_{u}-\frac{l_{u}}{k}, c_{d 33}^{(u)}=m_{u}-\frac{m_{u}^{s}}{k}, \widetilde{c}_{d 33}^{(u)}=m_{u}-\frac{m_{u}^{2}}{k},
$$

with $n_{u}^{s}=\sum_{i=1}^{t} n_{u i}^{2}, m_{u}=\sum_{i=1}^{t} m_{u i}, m_{u}^{s}=\sum_{i=1}^{t} m_{u i}^{2}, l_{u}=\sum_{i=1}^{t} n_{u i} m_{u i}$, denoting by $n_{u i}$ the number of plots in Block $u$ which receive Treatment $i$ and by $m_{u i}$ the number of times Treatment $i$ is preceded by itself in the Block $u$. It follows that:

$$
q_{d}(x)=\sum_{u=1}^{b} h_{d}^{(u)}(x)
$$

where

$h_{d}^{(u)}(x)=c_{d 33}^{(u)}-\frac{1}{t} \widetilde{c}_{d 33}^{(u)}+4\left(c_{d 13}^{(u)}-c_{d 33}^{(u)}+\frac{1}{t} \widetilde{c}_{d 33}^{(u)}\right) x+2\left(c_{d 11}^{(u)}+c_{d 12}^{(u)}-4 c_{d 13}^{(u)}+2 c_{d 33}^{(u)}-\frac{2}{t} \widetilde{c}_{d 33}^{(u)}\right) x^{2}$.

Two sequences of treatments in two blocks $u_{1}$ and $u_{2}$ are said to be equivalent if $\left(n_{u_{1}}^{s}, m_{u_{1}}, m_{u_{1}}^{s}, l_{u_{1}}\right)=\left(n_{u_{2}}^{s}, m_{u_{2}}, m_{u_{2}}^{s}, l_{u_{2}}\right)$, which is the case if one 
sequence is obtained from the other one by relabelling the treatments. So, for given $k$ and $t$, we can divide the set of all possible treatment sequences into $K$ equivalence classes of treatments. Since $n_{u}^{s}, m_{u}, m_{u}^{s}, l_{u}$ and $c_{d i j}^{(u)}$ are the same for all $u$ from one equivalent class $\ell$, we change the notation and write $n_{\ell}^{s}, m_{\ell}, m_{\ell}^{s}, l_{\ell}$ and $c_{d i j}(\ell)$ instead. We define:

$$
\begin{aligned}
h_{\ell}(x)= & \left(m_{\ell}-\frac{m_{\ell}^{s}}{k}-\frac{\delta_{\ell}}{t}\right)+\frac{4}{k}\left(m_{\ell}^{s}-l_{\ell}+\frac{k \delta_{\ell}}{t}\right) x \\
& +2\left[\left(k-m_{\ell}\right)+\frac{2}{k}\left(2 l_{\ell}-n_{\ell}^{s}-m_{\ell}^{s}\right)-\frac{2 \delta_{\ell}}{t}\right] x^{2}
\end{aligned}
$$

where $\delta_{\ell}=m_{\ell}\left(1-m_{\ell} / k\right)$. For a design $d$, we denote by $\pi_{d \ell}$ the proportion of blocks assigned to the class $\ell(1 \leq \ell \leq K)$. So, we have:

$$
q_{d}(x)=b \sum_{\ell=1}^{K} \pi_{d \ell} h_{\ell}(x)
$$

\subsection{Optimality conditions}

Our goal, in order to find an optimal configuration $d^{*}$, is to obtain a value $x^{*}$ and proportions $\pi_{d^{*}}^{\prime}=\left(\pi_{d^{*} 1}, \ldots, \pi_{d^{*} K}\right)$ such that:

$$
q_{d^{*}}\left(x^{*}\right)=\max _{\pi_{d}} q_{d}^{*} \text { with } q_{d}^{*}=\min _{x} q_{d}(x) .
$$

The following propositions characterizes universally optimal designs.

Proposition 6 (Kunert and Martin, 2000) Consider a symmetric design $d^{*} \in \Omega_{t, b, k}$ and a point $x^{*}$ such that the first derivative of $q_{d^{*}}$ is zero. 
If we have also:

$$
\forall \ell=1, \ldots, K, b h_{\ell}\left(x^{*}\right) \leq q_{d^{*}}^{*}
$$

then $d^{*}$ is universally optimal over $\Omega_{t, b, k}$.

The following proposition reduce to $k-1$ the number of equivalent classes that possibly appear in an optimal design. We denote by $\lfloor x\rfloor$ the integer part of $x$.

Proposition 7 If a symmetric design $d^{*} \in \Omega_{t, b, k}$ is universally optimal, then the treatment sequences present in the design necessarily satisfies:

1. all the periods receiving the same treatment are side by side in the sequence.

2. each treatment present in the sequence occurs $\lfloor k / v\rfloor$ or $\lfloor k / v\rfloor+1$ times, where $v$ is the number of different treatments present in the sequence. The number of treatments that occur $\lfloor k / v\rfloor+1$ times is $k-v\lfloor k / v\rfloor$ and the number of treatments that occurs $\lfloor k / v\rfloor$ times is $v(\lfloor k / v\rfloor+1)-k$.

Proof: The proof is given in Appendix B.

It is worth noting that for each value of $v$, there is only one equivalent class of treatment sequences that may appear in the optimal design. 


\subsection{Construction of optimal designs}

Consider a sequence $\ell$ present in an optimal design. From Proposition 7, a treatment $i$ in $\ell$ satisfies $m_{\ell i}=n_{\ell i}-1$. Moreover we have $v_{\ell}=k-m_{\ell}$. So $n_{\ell}^{s}=m_{\ell}^{s}+m_{\ell}+k, l_{\ell}=m_{\ell}^{s}+m_{\ell}$ and then:

$h_{\ell}(x)=\left(m_{\ell}-\frac{m_{\ell}^{s}}{k}-\frac{\delta_{\ell}}{t}\right)+\frac{4}{k}\left(\frac{k \delta_{\ell}}{t}-m_{\ell}\right) x+2\left[\left(k-m_{\ell}\right)\left(\frac{k-2}{k}\right)-\frac{2 \delta_{\ell}}{t}\right] x^{2}$,

with $\delta_{\ell}=m_{\ell}\left(1-m_{\ell} / k\right)$.

Therefore an optimal design for this one-dimensional problem is obtained using one class or a mixture of two different classes (see Kushner (1997)). The following method (for given $k$ and $t$ ) can be used in order to prove that a design $d^{*}$ is optimal.

- If the optimal design $d^{*}$ is constituted by one sequence of treatment $\ell_{1}$ (i.e. $\left.q_{d^{*}}(x)=b h_{\ell_{1}}(x)\right)$ :

- find $x^{*}$ that minimize $h_{\ell_{1}}$ and then the minimum $q_{d^{*}}^{*}$ of $q_{d^{*}}$,

- check that: $\forall \ell=1, \ldots, K, b h_{\ell}\left(x^{*}\right) \leq q_{d^{*}}^{*}$ (cf. Prop. 6).

- If the design $d^{*}$ is constituted by two different classes of treatments $\ell_{1}$ and $\ell_{2}\left(\right.$ i.e. $\left.q_{d^{*}}(x)=b\left(\pi_{d^{*} \ell_{1}} h_{\ell_{1}}(x)+\pi_{d^{*} \ell_{2}} h_{\ell_{2}}(x)\right)\right)$ :

- find an admissible intersection point $x^{*}$ according to the definition of Kushner (1997), i.e. $h_{\ell_{1}}\left(x^{*}\right)=h_{\ell_{2}}\left(x^{*}\right)$ and $\frac{\partial h_{\ell_{1}}}{\partial x}\left(x^{*}\right) \frac{\partial h_{\ell_{2}}}{\partial x}\left(x^{*}\right) \leq 0$, 
- find the proportions $\pi_{d^{*} \ell_{1}}^{*}$ and $\pi_{d^{*} \ell_{2}}^{*}$ in order to obtain: $\frac{\partial q_{d^{*}}}{\partial x}\left(x^{*}\right)=0$,

- use these proportions for finding the minimum $q_{d^{*}}^{*}=q_{d^{*}}\left(x^{*}\right)$ of $q_{d^{*}}$,

- check that: $\forall \ell=1, \ldots, K, b h_{\ell}\left(x^{*}\right) \leq q_{d^{*}}^{*}$ (cf. Prop. 6).

Note that the optimal proportions can be found by the following method.

$$
\frac{\partial q_{d^{*}}}{\partial x}\left(x^{*}\right)=0 \Longleftrightarrow b\left(\pi_{d^{*} \ell_{1}} \frac{\partial h_{\ell_{1}}}{\partial x}\left(x^{*}\right)+\pi_{d^{*} \ell_{2}} \frac{\partial h_{\ell_{2}}}{\partial x}\left(x^{*}\right)\right)=0 .
$$

Denote $a_{i}=\left(\partial h_{\ell_{i}} / \partial x\right)\left(x^{*}\right), i=1,2$. The optimal proportions are then:

$$
\pi_{d^{*} \ell_{1}}^{*}=\frac{a_{2}}{a_{2}-a_{1}} \text { and } \pi_{d^{*} \ell_{2}}^{*}=\frac{a_{1}}{a_{1}-a_{2}} .
$$

Now, we give optimal designs for several values of $k$. For $k=3,4$ we give explicit formulae. For $k=5, \ldots, 10$, we present numerical results.

1) The case $k=3$. From proposition 7 , an optimal design is constituted by one or two blocks in the following set of sequences:

\begin{tabular}{|c|c|c|c|c|}
\hline Sequence & $n_{\ell}^{s}$ & $m_{\ell}$ & $m_{\ell}^{s}$ & $l_{\ell}$ \\
\hline$\left[\begin{array}{lll}1 & 2 & 3\end{array}\right]$ & 3 & 0 & 0 & 0 \\
\hline$\left[\begin{array}{lll}1 & 1 & 2\end{array}\right]$ & 5 & 1 & 1 & 2 \\
\hline
\end{tabular}

So the functions $h_{\ell}$ are given by (we identify each class with its value of $m_{\ell}$ ):

$$
h_{0}(x)=2 x^{2} \text { and } h_{1}(x)=\frac{2}{3}\left(1-\frac{1}{t}\right)-\frac{4}{3}\left(1-\frac{2}{t}\right) x+\frac{4}{3}\left(1-\frac{2}{t}\right) x^{2} .
$$


It is impossible in that case to satisfy Proposition 6 using only one of these two sequences. So, we must find an admissible intersection point $x^{*}$. Some algebra shows that:

$$
x^{*}=\frac{\sqrt{(t-2)^{2}+(t-1)(t+4)}-(t-2)}{t+4} .
$$

The proportions in the optimal design $d^{*}$ are then:

$$
\pi_{d^{*} 0}^{*}=1-\pi_{d^{*} 1}^{*} \text { and } \pi_{d^{*} 1}^{*}=\frac{3 t}{t+4}\left(1-\frac{(t-2)}{\sqrt{t(2 t-1)}}\right) .
$$

\begin{tabular}{|c|c|c|c|c|c|c|}
\hline$t$ & 3 & 4 & 5 & 10 & 20 & $\infty$ \\
\hline Prop. [ [ $\left.\begin{array}{lll}1 & 2 & 3\end{array}\right]$ & 0.046 & 0.067 & 0.079 & 0.100 & 0.111 & 0.121 \\
\hline Prop. [ [ $\left.\begin{array}{lll}1 & 1 & 2\end{array}\right]$ & 0.954 & 0.933 & 0.921 & 0.899 & 0.889 & 0.879 \\
\hline
\end{tabular}

The following table gives the optimal proportions for several values of $t$.

The sequence [ $\left.\begin{array}{lll}1 & 1 & 2\end{array}\right]$ is predominating in this mixture. So it can be interesting in practice to use designs generated by only this sequence. The quality of such designs can be quantified by the classical $\Phi_{p}$ criteria. We know (see e.g. Druilhet, 2004) that when the information matrix is completely symmetric $\Phi_{p}$ does not depend on $p$. Thus we can derive the efficiency factor of a design $d \in \Omega_{t, b, 3}$ generated by one sequence $\ell$ :

$$
\operatorname{Eff}(d)=\frac{b h_{\ell}^{*}}{q_{d^{*}}\left(x^{*}\right)} \text { with } h_{\ell}^{*}=\min _{x} h_{\ell}(x)
$$


Numerical applications are given in the following table:

\begin{tabular}{|c|c|c|c|c|c|c|}
\hline$t$ & 3 & 4 & 5 & 10 & 20 & $\infty$ \\
\hline Eff. [ $\left[\begin{array}{lll}1 & 1 & 2\end{array}\right]$ & 0.989 & 0.985 & 0.982 & 0.976 & 0.974 & 0.971 \\
\hline
\end{tabular}

2) The case $k=4$. An optimal configuration is constituted by one or two blocks belonging to the following set of sequences:

\begin{tabular}{|c|c|c|c|c|}
\hline Sequence & $n_{\ell}^{S}$ & $m_{\ell}$ & $m_{\ell}^{s}$ & $l_{\ell}$ \\
\hline$\left[\begin{array}{llll}1 & 2 & 3 & 4\end{array}\right]$ & 4 & 0 & 0 & 0 \\
\hline$\left[\begin{array}{llll}1 & 1 & 2 & 3\end{array}\right]$ & 6 & 1 & 1 & 2 \\
\hline$\left[\begin{array}{llll}1 & 1 & 2 & 2\end{array}\right]$ & 8 & 2 & 2 & 4 \\
\hline
\end{tabular}

The functions $h_{\ell}$ are then given by:

$$
\begin{aligned}
& h_{0}(x)=4 x^{2}, h_{1}(x)=\frac{3}{4}\left(1-\frac{1}{t}\right)-\left(1-\frac{3}{t}\right) x+3\left(1-\frac{1}{t}\right) x^{2}, \\
& h_{2}(x)=\left(\frac{3}{2}-\frac{1}{t}\right)-2\left(1-\frac{2}{t}\right) x+2\left(1-\frac{2}{t}\right) x^{2} .
\end{aligned}
$$

For any $t$, the minimum of $h_{2}$ is obtained for $x^{*}=0.5$ and satisfies the conditions of proposition 6 . So the optimal design is generated by the sequence [ $\left.\begin{array}{llll}1 & 1 & 2 & 2\end{array}\right]$. As an example we can consider, for $t=4$, the optimal design such that:

$$
D=\left[\begin{array}{cccccccccccc}
1 & 2 & 1 & 3 & 1 & 4 & 2 & 3 & 2 & 4 & 3 & 4 \\
1 & 2 & 1 & 3 & 1 & 4 & 2 & 3 & 2 & 4 & 3 & 4 \\
2 & 1 & 3 & 1 & 4 & 1 & 3 & 2 & 4 & 2 & 4 & 3 \\
2 & 1 & 3 & 1 & 4 & 1 & 3 & 2 & 4 & 2 & 4 & 3
\end{array}\right] \in \Omega_{4,12,4}
$$


Note that, by circularity, the design obtained by taking away one out of every two columns is universally optimal over all the designs in $\Omega_{4,6,4}$.

3) The case $k=5$. The optimal design is generated by the following mixtures:

\begin{tabular}{|c|c|c|c|c|c|c|}
\hline$t$ & 3 & 4 & 5 & 10 & 20 & $\infty$ \\
\hline Prop. $\left[\begin{array}{lllll}1 & 1 & 2 & 2 & 3\end{array}\right]$ & 0.167 & 0.250 & 0.300 & 0.400 & 0.450 & 0.500 \\
\hline Prop. $\left[\begin{array}{lllll}1 & 1 & 1 & 2 & 2\end{array}\right]$ & 0.833 & 0.750 & 0.700 & 0.600 & 0.550 & 0.500 \\
\hline
\end{tabular}

The efficiencies of designs generated by only one sequence are:

\begin{tabular}{|c|c|c|c|c|c|c|}
\hline$t$ & 3 & 4 & 5 & 10 & 20 & $\infty$ \\
\hline Eff. [ $\left[\begin{array}{lllll}1 & 1 & 2 & 2 & 3\end{array}\right]$ & 0.844 & 0.895 & 0.918 & 0.949 & 0.961 & 0.970 \\
\hline Eff. [ [ $\left.\begin{array}{lllll}1 & 1 & 1 & 2 & 2\end{array}\right]$ & 0.984 & 0.977 & 0.972 & 0.963 & 0.959 & 0.955 \\
\hline
\end{tabular}

It can be observed that the design generated by [ $\left.\begin{array}{llllll}1 & 1 & 1 & 2 & 2\end{array}\right]$ is more efficient than the one generated by $\left[\begin{array}{lllll}1 & 1 & 1 & 2 & 2\end{array}\right]$ for $t>18$.

4) The case $k=6$. The optimal design is generated by the following mixtures:

\begin{tabular}{|c|c|c|c|c|c|c|}
\hline$t$ & 3 & 4 & 5 & 6 & 7 & $\geq 8$ \\
\hline Prop. [ [ $\left.\begin{array}{llllll}1 & 1 & 2 & 2 & 3 & 3\end{array}\right]$ & 0.400 & 0.628 & 0.775 & 0.878 & 0.954 & 1.000 \\
\hline Prop. [ [ $\left.\begin{array}{llllll}1 & 1 & 1 & 2 & 2 & 2\end{array}\right]$ & 0.600 & 0.372 & 0.225 & 0.122 & 0.046 & \\
\hline
\end{tabular}


The efficiencies of designs generated by only one sequence are:

\begin{tabular}{|c|c|c|c|c|c|c|}
\hline$t$ & 3 & 4 & 5 & 6 & 7 & $\geq 8$ \\
\hline Eff. [ [ $\left.\begin{array}{llllll}1 & 1 & 2 & 2 & 3 & 3\end{array}\right]$ & 0.962 & 0.989 & 0.997 & 0.999 & 0.999 & 1.000 \\
\hline Eff. [ [ $\left.\begin{array}{llllll}1 & 1 & 1 & 2 & 2 & 2\end{array}\right]$ & 0.962 & 0.942 & 0.930 & 0.922 & 0.917 & \\
\hline
\end{tabular}

We note that the sequence [ $\left[\begin{array}{llllll}1 & 1 & 2 & 2 & 3 & 3\end{array}\right]$ is always more efficient than the sequence [ $\left[\begin{array}{llllll}1 & 1 & 1 & 2 & 2 & 2\end{array}\right]$. It also generates an optimal design when $t \geq 8$.

5) The case $k=7$. The optimal design is generated by the following mixtures:

\begin{tabular}{|c|c|c|}
\hline$t$ & 3 & $\geq 4$ \\
\hline Prop. $\left[\begin{array}{lllllll}1 & 1 & 1 & 2 & 2 & 3 & 3\end{array}\right]$ & 0.682 & 1.000 \\
\hline Prop. $\left[\begin{array}{lllllll}1 & 1 & 1 & 1 & 2 & 2 & 2\end{array}\right]$ & 0.318 & \\
\hline
\end{tabular}

The efficiencies of designs generated by only one sequence are:

\begin{tabular}{|c|c|c|}
\hline$t$ & 3 & $\geq 4$ \\
\hline Eff. [ [ $\left.\begin{array}{lllllll}1 & 1 & 1 & 2 & 2 & 3 & 3\end{array}\right]$ & 0.994 & 1.000 \\
\hline Eff. [ $\left[\begin{array}{lllllll}1 & 1 & 1 & 2 & 2 & 3 & 3\end{array}\right]$ & 0.938 & \\
\hline
\end{tabular}

We note that he sequence [ $\left[\begin{array}{llllll}1 & 1 & 1 & 2 & 2 & 3\end{array}\right]$ ] is always better than the sequence

$\left[\begin{array}{llllll}1 & 1 & 1 & 1 & 2 & 2\end{array}\right]$ and generates the optimal design when $t \geq 4$.

6) The case $k=8,9,10$. We find that the optimal design is generated, for 
every $t$, by only one sequence. These optimal sequences are given by:

\begin{tabular}{|c|c|c|c|}
\hline$t$ & 8 & 9 & 10 \\
\hline Sequence & {$\left[\begin{array}{llllllll}1 & 1 & 1 & 2 & 2 & 2 & 3 & 3\end{array}\right]$} & {$\left[\begin{array}{lllllllll}1 & 1 & 1 & 2 & 2 & 2 & 3 & 3 & 3\end{array}\right]$} & {$\left[\begin{array}{llllllllll}1 & 1 & 1 & 1 & 2 & 2 & 2 & 3 & 3 & 3\end{array}\right]$} \\
\hline
\end{tabular}

\section{Models with period effects}

We consider the model:

$$
y_{i j}=\alpha_{j}+\beta_{i}+\tau_{d(i, j)}+\lambda_{d(i, j-1)}+\chi_{d(i, j-1) d(i, j)}+\varepsilon_{i j}
$$

where $\alpha_{j}$ is the effects of the period $j$. We denote by $A$ the corresponding incidence matrix. A balanced design is a design such that all the sequences belonging to the same equivalent class appear equally often. This notion is slightly more restrictive than the notion of symmetric design defined in Section 4.1 but correspond to the design generated by one sequence or a mixture of sequences, considering all the treatment permutations.

Proposition 8 A balanced design which is universally optimal for total effects under Model (1) is also universally optimal under Model (11).

Proof: The proof is a direct consequence of the following lemma.

Lemma 9 For a balanced design, the information matrix for the total effects is the same under model (1) and (11) 
Proof: Let $d$ be a balanced design. The difficulty is that interaction and period effects are not orthogonal. Write $\tilde{\theta}^{\prime}=(\tau|\lambda| \chi \mid \alpha)$ and $\tilde{C}_{d}[\tilde{\theta}]$ the corresponding information matrix. We want to prove that $\tilde{C}_{d}\left[\tilde{K}^{\prime} \tilde{\theta}\right]=C_{d}\left[K^{\prime} \theta\right]$ where $\tilde{K}^{\prime}=\left(I_{t}\left|I_{t}\right| I_{t} \mid 0\right)$. We write:

$$
\tilde{C}_{d}[\tilde{\theta}]=\left(\begin{array}{cc}
C_{d}[\theta] & C_{d 12} \\
C_{d 21} & C_{d 22}
\end{array}\right)
$$

where $C_{d 12}^{\prime}=\left(0|0| D_{d}^{\prime}\right)$ with $D_{d}=\left(p_{d 1} \mathbb{1}_{t}\left|p_{d 2} \mathbb{1}_{t}\right| \ldots \mid p_{d b} \mathbb{1}_{t}\right)$. The real $p_{d j}$ depends only on the number of times a treatment is preceded by itself on period $j$. Note that the usual orthogonality condition (see Kunert, 1983) between interaction and period effects is $D_{d}=0$ which is not the case here. The key point of the proof is that

$$
Q_{t} D_{d}=0
$$

Denote by $\tilde{L}_{d}^{*}$ a matrix such that $\tilde{C}_{d}\left[\tilde{K}^{\prime} \tilde{\theta}\right]=\tilde{L}_{d}^{*}{ }^{\prime} \tilde{C}_{d}[\tilde{\theta}] \tilde{L}_{d}^{*}$ and $\tilde{L}_{d}^{*}{ }^{\prime} \tilde{K}=I_{t}$. We write $\tilde{L}_{d}^{* \prime}=\left(M_{d}^{* \prime} \mid N_{d}^{* \prime}\right)$ where $M_{d}$ is a $(3 t \times t)$ matrix and $N_{d}^{*}$ is a $(b \times b)$ matrix. It is easy to see that $C_{d}\left[K^{\prime} \theta\right] \mathbb{1}_{t}=0$ and, since $\tilde{C}_{d}\left[\tilde{K}^{\prime} \tilde{\theta}\right] \leq C_{d}\left[K^{\prime} \theta\right]$, we have $\tilde{C}_{d}\left[\tilde{K}^{\prime} \tilde{\theta}\right] \mathbb{1}_{t}=0$. So, $C_{d}\left[\tilde{K}^{\prime} \tilde{\theta}\right]=Q_{t} \tilde{C}_{d}\left[\tilde{K}^{\prime} \tilde{\theta}\right] Q_{t}$. Therefore, $\tilde{L}^{*}$ can be chosen equal to $\left(M_{d}^{*} Q_{t} \mid N_{d}^{*} Q_{t}\right)$. For any permutation $\sigma$, we have $\left(I_{3} \otimes\right.$ $\left.P_{\sigma}\right) C_{d 12}=C_{d 12}$ and therefore, similarly to Proposition 1, it can be shown that the three $(t \times t)$ blocks of $M_{d}^{*}$ can be chosen completely symmetric. So, $\tilde{L}_{d}^{*}{ }^{\prime}$ can be chosen equal to $\left(x_{1}^{*} Q_{t}\left|x_{2}^{*} Q_{t}\right|\left(1-\left(x_{1}^{*}+x_{2}^{*}\right)\right) Q_{t} \mid N^{*}\right)$. Put 
$\tilde{L}^{\prime}=\left(M^{\prime} \mid N^{\prime}\right)$ with $M^{\prime}=\left(x_{1} Q_{t}\left|x_{2} Q_{t}\right|\left(1-\left(x_{1}+x_{2}\right) Q_{t}\right)\right.$. By (12), we have $M^{\prime} C_{d 12}=0$ and then

$$
\tilde{C}_{d}\left[\tilde{K}^{\prime} \tilde{\theta}\right]=\min _{x_{1}, x_{1}, N} \tilde{L}^{\prime} \tilde{C}[\tilde{\theta}] \tilde{L}=\min _{x_{1}, x_{1}, N}\left(M^{\prime} C_{d}[\theta] M+N^{\prime} C_{d 12} N\right)
$$

Since $x_{1}, x_{2}$ and $N$ vary freely, $N$ can be chosen equal to 0 and therefore, from Example 4 of Section 3.2 and the constraint on $y$ obtained in Lemma $5, M^{*}{ }^{\prime} \tilde{C}[\tilde{\theta}] M^{*}=L^{*}{ }^{\prime} C_{d}[\theta] L^{*}$. The result follows.

\section{Appendix A}

We give here the proof of Proposition 1.

Step 1: We show that the set $E$ of matrices $L$ that minimize $L^{\prime} C[\alpha] L$ under the constraint $L^{\prime} K=I_{t}$ is an affine subspace: consider $L_{a}$ and $L_{b}$ in $E$ with $L_{a} \neq L_{b}$. It is sufficient to prove that $L_{\gamma}=\gamma L_{a}+(1-\gamma) L_{b}$ belongs to $E$ for any real $\gamma$. We have $L_{\gamma}^{\prime} K=I_{t}$ and

$$
L_{\gamma}^{\prime} C[\alpha] L_{\gamma}=\gamma^{2} L_{a}^{\prime} C[\alpha] L_{a}+(1-\gamma)^{2} L_{b}^{\prime} C[\alpha] L_{b}+\gamma(1-\gamma)\left(L_{a}^{\prime} C[\alpha] L_{b}+L_{b}^{\prime} C[\alpha] L_{a}\right)
$$

This quadratic function w.r.t. $\gamma$ admits two minima relative to the Loewner ordering at $\gamma=0$ and $\gamma=1$ and therefore it is constant. So, $L_{\gamma}$ minimizes $L^{\prime} C[\alpha] L$ for any $\gamma \in \mathbb{R}$. 
Step 2: Consider a permutation $\sigma$ on $\{1, \ldots, t\}$ and denote by $P_{\sigma}$ the corresponding permutation matrix. We want to prove that if $L^{*} \in E$, so does $L_{\sigma}^{*}=\left(I_{3} \otimes P_{\sigma}^{\prime}\right) L^{*} P_{\sigma}$. Since $C_{i j}$ and $K_{i}$ are completely symmetric:

$$
\begin{array}{ll} 
& \left(I_{3} \otimes P_{\sigma}\right) C[\alpha]\left(I_{3} \otimes P_{\sigma}^{\prime}\right)=C[\alpha], \\
\text { and } \quad & \left(I_{3} \otimes P_{\sigma}^{\prime}\right) K=K P_{\sigma} .
\end{array}
$$

Moreover,

$$
\begin{aligned}
C\left[K^{\prime} \alpha\right] & =\min _{L \in \mathbb{R}^{3 t \times t}: L^{\prime} K=I_{t}} L^{\prime} C[\alpha] L, \\
& =\min _{L \in \mathbb{R}^{3 t \times t}: L^{\prime} K=I_{t}} L^{\prime}\left(I_{t} \otimes P_{\sigma}\right) C[\alpha]\left(I_{t} \otimes P_{\sigma}^{\prime}\right) L \quad(\text { by }(13)), \\
& =P_{\sigma}\left(\min _{L \in \mathbb{R}^{3 t \times t}: L^{\prime} K=I_{t}} P_{\sigma}^{\prime} L^{\prime}\left(I_{t} \otimes P_{\sigma}\right) C[\alpha]\left(I_{t} \otimes P_{\sigma}^{\prime}\right) L P_{\sigma}\right) P_{\sigma}^{\prime} .
\end{aligned}
$$

Put $L_{\sigma}=\left(I_{3} \otimes P_{\sigma}^{\prime}\right) L P_{\sigma}$. By (14), $L_{\sigma}^{\prime} K=I_{t}$. Since, $L \leftrightarrow L_{\sigma}$ is a one to one mapping, we have:

$$
C\left[K^{\prime} \alpha\right]=P_{\sigma}\left(\min _{L_{\sigma} \in \mathbb{R}^{3+\times t}: L_{\sigma}^{\prime} K=I_{t}} L_{\sigma}^{\prime} C[\alpha] L_{\sigma}\right) P_{\sigma}^{\prime}=P_{\sigma} C\left[K^{\prime} \alpha\right] P_{\sigma}^{\prime} .
$$

Since Eq. (15) holds for any permutation $\sigma, C\left[K^{\prime} \theta\right]$ is completely symmetric. So,

$$
C\left[K^{\prime} \alpha\right]=\min _{L_{\sigma} \in \mathbb{R}^{3 t \times t}: L_{\sigma}^{\prime} K=I_{t}} L_{\sigma}^{\prime} C[\alpha] L_{\sigma}
$$

and therefore $C\left[K^{\prime} \alpha\right]=L_{\sigma}^{* \prime} C[\alpha] L_{\sigma}^{*}$.

Step 3: If $L^{*} \in E$ then, by step 1 and $2, \bar{L}^{*}=\frac{1}{t !} \sum_{\sigma} L_{\sigma}^{*}$ also belongs to $E$. By construction, $\bar{L}_{i}^{*}$ is completely symmetric and the proof is complete. 


\section{Appendix B}

We prove here Proposition 7. From Eq. (10), we have $h_{\ell}(x)=A_{\ell}+B_{\ell} x+$

$C_{\ell} x^{2}$ where: $A_{\ell}=m_{\ell}-\frac{m_{\ell}^{s}}{k}-\frac{\delta_{\ell}}{t}, B_{\ell}=\frac{4}{k}\left(m_{\ell}^{s}-l_{\ell}+\frac{k \delta_{\ell}}{t}\right)$ and $C_{\ell}=$ $2\left[\left(k-m_{\ell}\right)+\frac{2}{k}\left(2 l_{\ell}-n_{\ell}^{s}-m_{\ell}^{s}\right)-\frac{2 \delta_{\ell}}{t}\right]$. We denote by $v_{\ell}$ the number of treatments present in the sequence $\ell$. For any treatment $i$ in $\ell$, we have:

$$
n_{\ell i} \geq m_{\ell i}+1
$$

with equality if and only if treatment $i$ occurs in consecutive periods. In general, each treatment appears in the sequence $\ell$ in several groups of consecutive periods. The number of such groups is $\gamma_{i}=n_{\ell i}-m_{\ell i}$. From $\ell$, we construct a new sequence $\widetilde{\ell}$ as follows: in each group of consecutive periods receiving the same treatment, we replace the treatment that occurs in this group by a new treatment, such that in the new sequence $\widetilde{\ell}$, each treatment have only one group. For example $\ell=(1,1,2,2,2,1,3,2,2)$ gives $\tilde{\ell}=(1,1,2,2,2,4,3,5,5)$. We have $m_{\tilde{\ell}}=m_{\ell}$ and $v_{\tilde{\ell}}=\gamma=k-m_{\ell}$, where $\gamma=\sum_{i} \gamma_{i}$ is the number of different groups in $\ell$.

Lemma 10 If $d^{*}$ is optimal, then $x^{*}$ that minimizes $q_{d^{*}}(x)$ is positive.

Proof. From Lemma 3 we know that $h_{\ell}(x)$ is convex, then $C_{\ell} \geq 0$. So it is sufficient to show that $B_{\ell} \leq 0$. If $C_{\ell}=0$ then $B_{\ell}=0$ because we know 
that $h_{\ell}(x)$ admits a minimum. In that case, any $x$ is a minimum and can be chosen positive. We assume now that $C_{\ell}>0$. By Eq. 16, we have $l_{\ell}=\sum_{i} m_{\ell i} n_{\ell i} \geq m_{\ell}+m_{\ell}^{s}$ and then

$$
B_{\ell} \leq \frac{4}{k t} m_{\ell}\left(k-m_{\ell}-t\right)
$$

with equality if and only if the occurrences of the treatments present in the sequence are placed side by side. In that case a sequence $\tilde{\ell}$ satisfies $k-m_{\tilde{\ell}}=$ $v_{\widetilde{\ell}}$ and, since $t \geq v_{\widetilde{\ell}}, B_{\widetilde{\ell}} \leq 0$. Consider now a general sequence $\ell$ and denote by $\tilde{\ell}$ its associated sequence with side-by-side treatments. Since $m_{\tilde{\ell}}=m_{\ell}$ we have also $\delta_{\ell}=\delta_{\tilde{\ell}}$ and then $B_{\ell} \leq B_{\tilde{\ell}}$ if and only if $\left(l_{\ell}-m_{\ell}^{s}\right) \geq\left(l_{\tilde{\ell}}-m_{\tilde{\ell}}^{s}\right)$. But:

$$
l_{\ell}-m_{\ell}^{s}=\sum_{i=1}^{v_{\ell}} m_{\ell i}\left(n_{\ell i}-m_{\ell i}\right) \geq \sum_{i=1}^{v_{\ell}} m_{\ell i}=m_{\ell}=\sum_{i=1}^{v_{\widetilde{\ell}}} m_{\widetilde{\ell} i}\left(n_{\widetilde{\ell} i}-m_{\widetilde{\ell}}\right)
$$

with equality if and only if $\ell=\tilde{\ell}$. So we have for every sequence $\ell$ : $B_{\ell} \leq$ $B_{\widetilde{\ell}} \leq 0$

The proof of the Proposition 7 is given below.

Step 1: Let $\ell$ be a sequence containing $v_{\ell}$ different treatments numbered $1, \ldots, v_{\ell}$ and $\tilde{\ell}$ the associated sequence defined above. We want to prove that $h_{\tilde{\ell}}(x)>h_{\ell}(x)$ for all $x \geq 0$ and $\ell \neq \tilde{\ell}$. The idea is to show that $A_{\ell}<A_{\tilde{\ell}}$, $B_{\ell}<B_{\widetilde{\ell}}$ and $C_{\ell}<C_{\widetilde{\ell}}$. Note that $m_{\ell}=m_{\widetilde{\ell}}$ so $\delta_{\ell}=\delta_{\widetilde{\ell}}$. Then: 
- We show that $C_{\ell}<C_{\tilde{\ell}}$. It is equivalent to show that $\left(n_{\ell}^{s}-2 l_{\ell}+m_{\ell}^{s}\right)>$ $\left(n_{\widetilde{\ell}}^{s}-2 l_{\widetilde{\ell}}+m_{\widetilde{\ell}}^{s}\right)$. Since $n_{\widetilde{\ell} i}=m_{\widetilde{\ell} i}+1$ we have:

$n_{\ell}^{s}-2 l_{\ell}+m_{\ell}^{s}=\sum_{i=1}^{v_{\ell}}\left(n_{\ell i}-m_{\ell i}\right)^{2}=\sum_{i=1}^{v_{\ell}} \gamma_{i}^{2} \geq \sum_{i=1}^{v_{\ell}} \gamma_{i}=k-m_{\ell}=\sum_{i=1}^{v_{\widetilde{\ell}}}\left(n_{\widetilde{\ell} i}-m_{\widetilde{\ell} i}\right)^{2}$

with equality if and only if $\ell=\tilde{\ell}$. The result follows.

- From Lemma 10, we have $B_{\ell}<B_{\tilde{\ell}}$.

- We show that $A_{\ell}<A_{\tilde{\ell}}$. It is equivalent to show that $m_{\ell}^{s}>m_{\tilde{\ell}}^{s}$. This follows from the fact that $\sum_{i} m_{\ell i}=\sum_{i} m_{\widetilde{\ell} i}$ and, for all $i, m_{\ell i} \geq m_{\widetilde{\ell} i}$ with equality if and only if $\ell=\tilde{\ell}$.

Step 2: Consider the set $\mathcal{L}$ of sequences $\ell$ having the same number $v_{\ell}=v$ of distinct treatments and such that $\ell=\tilde{\ell}$. For a sequence $\ell \in \mathcal{L}, n_{\ell i}=m_{\ell i}-1$ and $m_{\ell}=k-v$. So we have for every $\ell_{1}, \ell_{2} \in \mathcal{L}$ (see Section 5.2):

$$
h_{\ell_{1}}(x)-h_{\ell_{2}}(x)=\frac{1}{k}\left(m_{\ell_{2}}^{s}-m_{\ell_{1}}^{s}\right) .
$$

If there exists a sequence $\ell^{*} \in \mathcal{L}$ such that $h_{\ell^{*}}(x)-h_{\ell}(x)>0$ (for every $\left.\ell \neq \ell^{*}\right)$ then only this sequence can belong to the optimal design. Such a sequence minimizes $m_{\ell}^{s}=\sum_{i=1}^{v_{\ell}} m_{\ell i}^{2}$ under the constraint $\sum_{i=1}^{v_{\ell}} m_{\ell i}=m_{\ell}$. So, it is well known that the $m_{\ell^{*} i}$ or equivalently the $n_{\ell^{*} i}\left(i=1, \ldots, v_{\ell^{*}}\right)$ must be as equal as possible: if $k / v_{\ell^{*}}$ is an integer, a treatment present 
in $\ell^{*}$ occurs $k / v_{\ell^{*}}$ times; otherwise, a treatment present in $\ell^{*}$ occurs either $\left\lfloor k / v_{\ell^{*}}\right\rfloor$ or $\left\lfloor k / v_{\ell^{*}}\right\rfloor+1$ times in $\ell^{*}$

\section{References}

Afsarinejad, K. and Hedayat, A. S. (2002). Repeated measurements designs for a model with self and simple mixed carryover effects. J. Statist. Plann. Inference, 106(1-2):449-459. Experimental design and related combinatorics.

Bailey, R. A. and Druilhet, P. (2004). Optimality of neighbor-balanced designs for total effects. Ann. Statist., 32(4):1650-1661.

Druilhet, P. (2004). Conditions for optimality in experimental designs. Linear Algebra Appl., 388:147-157.

Gaffke, N. (1987). Further characterizations of design optimality and admissibility for partial parameter estimation in linear regression. Ann. Statist., 15(3):942-957.

Kiefer, J. (1975). Construction and optimality of generalized Youden designs. In A survey of statistical design and linear models (Proc. Internat. Sympos., Colorado State Univ., Ft. Collins, Colo., 1973), pages 333-353. North-Holland, Amsterdam. 
Kunert, J. (1983). Optimal design and refinement of the linear model with applications to repeated measurements designs. Ann. Statist., 11(1):247257.

Kunert, J. and Martin, R. J. (2000). On the determination of optimal designs for an interference model. Ann. Statist., 28(6):1728-1742.

Kunert, J. and Stufken, J. (2002). Optimal crossover designs in a model with self and mixed carryover effects. J. Amer. Statist. Assoc., 97(459):898906.

Kushner, H. B. (1997). Optimal repeated measurements designs: the linear optimality equations. Ann. Statist., 25(6):2328-2344.

Pukelsheim, F. (1993). Optimal design of experiments. Wiley Series in Probability and Mathematical Statistics: Probability and Mathematical Statistics. John Wiley \& Sons Inc., New York. A Wiley-Interscience Publication. 
CREST-ENSAI

et IRMAR UMR CNRS 6625

Campus de Ker Lann

BP37203

35172 BRUZ CEDEX , France

E-mail: druilhet@ensai.fr
Laboratoire de mathématiques

appliquées UMR CNRS 5142

Université de Pau

avenue de l'Université BP 1155

64013 PAU CEDEX, France

E-mail: walter.tinsson@univ-pau.fr 